\title{
Design and application of accelerated life test of grating ruler based on step stress loading
}

\author{
Jili Wang ${ }^{1,2, *}$, Qingyu $\mathrm{Li}^{1,2}$, Xiaocui Zhu ${ }^{1,2}$, Cheng $\mathrm{Gao}^{3}, \mathrm{Yi} \mathrm{Li}^{1,2}$, Guofa $\mathrm{Li}^{1,2}$, and \\ Jialong $\mathrm{He}^{1,2}$ \\ ${ }^{1}$ Key Laboratory of CNC Equipment Reliability, Ministry of Education, Jilin University, Changchun, \\ Jilin, China. \\ ${ }^{2}$ School of Mechanical and Aerospace Engineering, Jilin University, Changchun, Jilin, China. \\ ${ }^{3}$ Avic Manufacturing Technology Institute, Bejing, China.
}

\begin{abstract}
Combined with the actual project, a grating ruler accelerated life test device is designed, which can simulate the actual loads, including temperature, humidity and speed stress. An accelerated life test scheme based on stepped stress loading is proposed, and 6 grating rulers are tested based on time-censored test method. An accelerated life model based on Weibull distribution used for evaluating the lifetime is established. Related experimental techniques also can be typical application cases for innovative practical teaching.
\end{abstract}

Grating ruler is a high-precision linear displacement measurement sensor which is used in closed-loop feedback link of CNC machine tool control system. It is one of the key parts that affect the reliability of CNC machine tool. However,compared with foreign advanced products, domestic grating rulers have frequent failures and low reliability levels have become the the bottleneck of its industry development. Therefore, to carry out the accelerated life test of the grating ruler, which aims at quickly stimulating and exposing the weak links of its reliability, and then optimizing the reliability of its design, is of great significance to the reliability improvement of the domestic grating ruler and the domestic high-end CNC machine tool.

\section{Design of accelerated life test system for grating ruler}

The basic requirements of the accelerated life test system of grating ruler are as follows: the type of loading stress is the same as the actual working condition, which ensures that the failure mechanism doesn't change. The test stress must be within the normal working stress range of the grating ruler and the test system. The test system can monitor the running state of the grating ruler in real time to prevent unrelated failures. Based on the above principles, the stress loading system, the condition monitoring system and the precision measurement scheme of the grating rule are designed.

\footnotetext{
* Corresponding author: wangjili1100@,126.com
} 


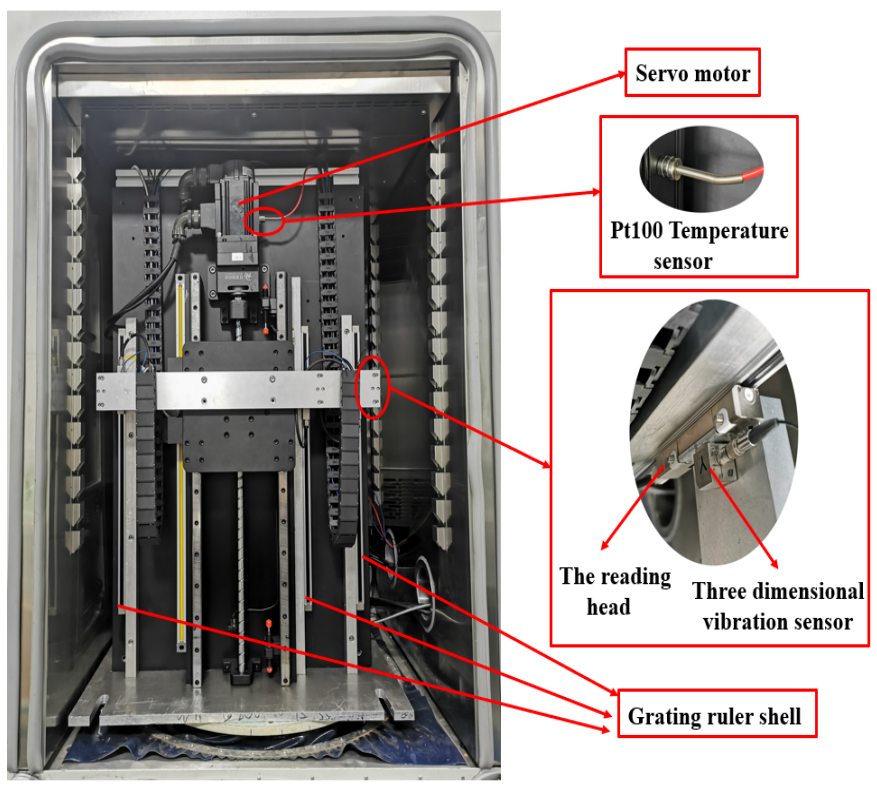

Fig. 1. Hardware system for accelerated life test of grating ruler.

\subsection{Stress loading system}

The stress loading system of the grating ruler accelerated life test includes the servo drive platform of grating ruler, temperature and humidity comprehensive test chamber and control system. It can simulate the actual working conditions of the grating ruler and realize the comprehensive stress loading such as speed, temperature and humidity.

The grating ruler servo drive platform is used to apply velocity stress to the tested grating ruler. As shown in Figure 1, the servo drive platform is a symmetrical vertical structure ${ }^{[1]}$, with the same structure on both sides. Three tested grating rulers are driven by a servo motor on each side at the same time. The maximum sample size of the tested grating ruler which can be installed is 6 . The tested grating ruler is installed on the mounting plate through the adjustable screw. Only one grating ruler can be installed on each mounting plate, and the different structure of mounting plates needs to be processed according to different models of grating rulers. The mounting plate is fixed on the bottom plate of the servo drive platform, so that the grating ruler remains static during the test. The reading head of the tested grating ruler is installed on the aluminum angle bearing. Only one reading head can be installed on each aluminum angle bearing, and the different structure of aluminum angle bearing needs to be processed according to different models of grating rulers. The three aluminum angle bearing are installed on the linkage aluminum plate through the adjustable screw holes. The servo motor drives the linkage aluminum plate to make the reading heads of the grating rulers perform reciprocating vertical linear motion,so as to realize the speed stress loading of the tested grating ruler. 


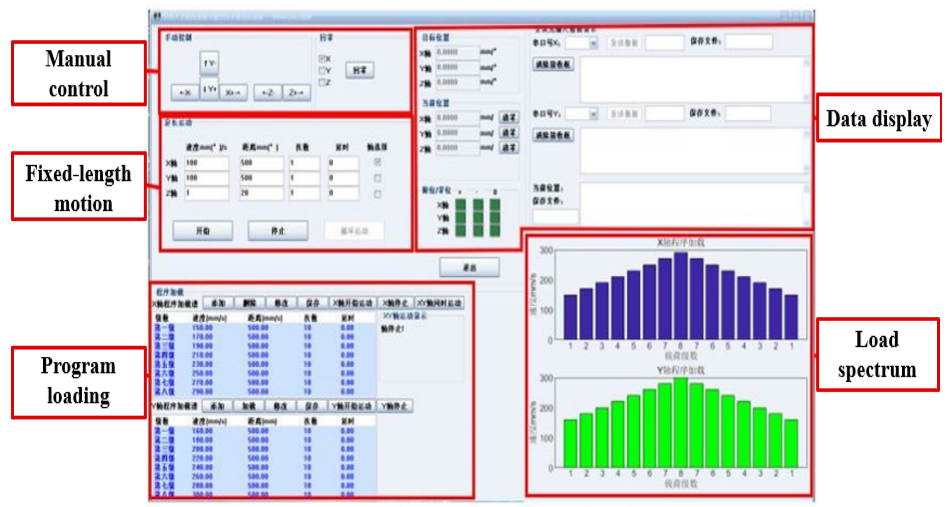

Fig. 2. Speed loading control program interface.

The control program of the servo drive platform is programed based on Microsoft Visual Studio software. Its human-computer interface is shown in Figure 2. The program can realize three motion control modes: manual control,fixed-length motion control and program loading control. The manual control mode is mainly used for commissioning test table. The fixed-length motion control mode can control the grating ruler to run cyclically at a fixed speed which is suitable for constant-stress test. The program loading control mode can control the tested grating ruler to run cyclically according to the compiled velocity stress loading spectrum.

The temperature and humidity loading control can be realized by operating the control program of the temperature and humidity comprehensive test box, and the control interface is shown in Figure 3. The temperature value, humidity value, temperature slope, humidity slope and running time can be set through this interface. Among them, the temperature and humidity values are the stress target values at final stable loading of the test chamber,and the loading time is the set operation time. The slope of temperature and humidity is the rate of change and the function of timing operation will start when the stress values of temperature and humidity change to the target value. During the accelerated life test of the grating ruler,the slope value of temperature and humidity should be as small as possible to prevent the impact of temperature and humidity change on the reliability of the grating ruler and test system.

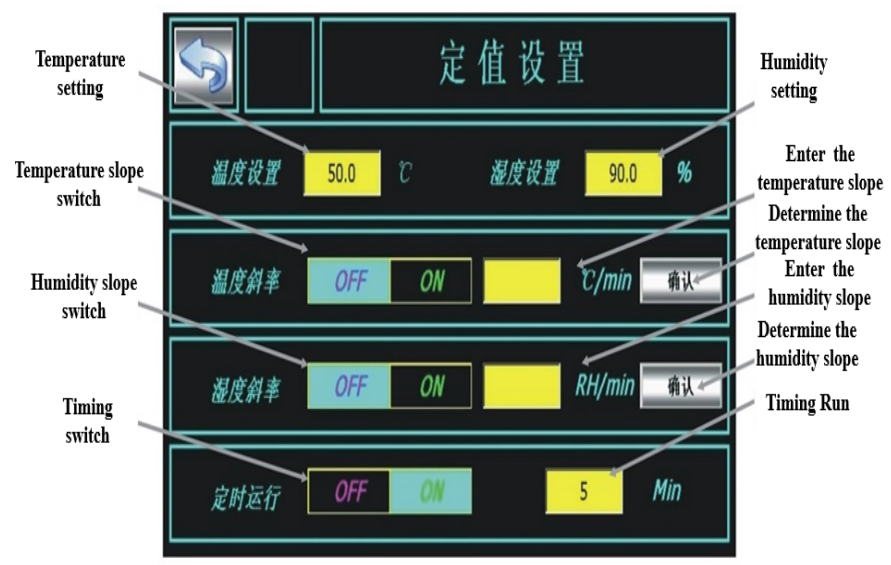

Fig. 3. Control program interface of temperature and humidity comprehensive test box. 


\subsection{Condition monitoring system}

The condition monitoring system of test table is programed with the LabVIEW graphical programming language, which is mainly used to monitor the operation status of the tested grating ruler and the test system in real time. The system can monitor vibration and voltage values of the reading head of the tested grating ruler during the test. If the vibration or voltage values of the grating ruler exceed pre-set securitythreshold, the monitoring system will immediately alarm and execute the emergency stop command to stop applying speed stress so as to protect the grating rulers and the test system. As shown in Figure 4, the waveforms of vibration and voltage signals of each grating ruler during the test are respectively displayed in the vibration and voltage monitoring modules in real time. Considering that the grating ruler is a product with high reliability and long life, its reliability is usually higher than the servo drive platform with more complex system structure. The servo drive platform is likely to fail before the grating ruler during the accelerated life test,so it is very important to monitor the reliability status of the test system. The most intuitive parameter is the motor temperature for the fault of the servo drive platform. For example, the motor surface temperature will rise due to the motor stall or the wear of the transmission parts, while the motor shutdown caused by the fault of cable and interface will lead to the decreasing of motor surface temperature. Therefore, it is necessary to monitor the temperature value of two servo motors in real time during the test, and monitor the internal ambient temperature of the test box as a reference. The three temperature values are displayed in the temperature monitoring module in real time in Fig. 4. The sampling frequency, unit and other parameters of the vibration acquisition card and voltage acquisition card can be set in the configuration interface of the real-time condition monitoring system. In addition, the above monitored data of temperature, vibration and voltage will be continuously stored by the condition monitoring system to facilitate data subsequent processing and analysis.

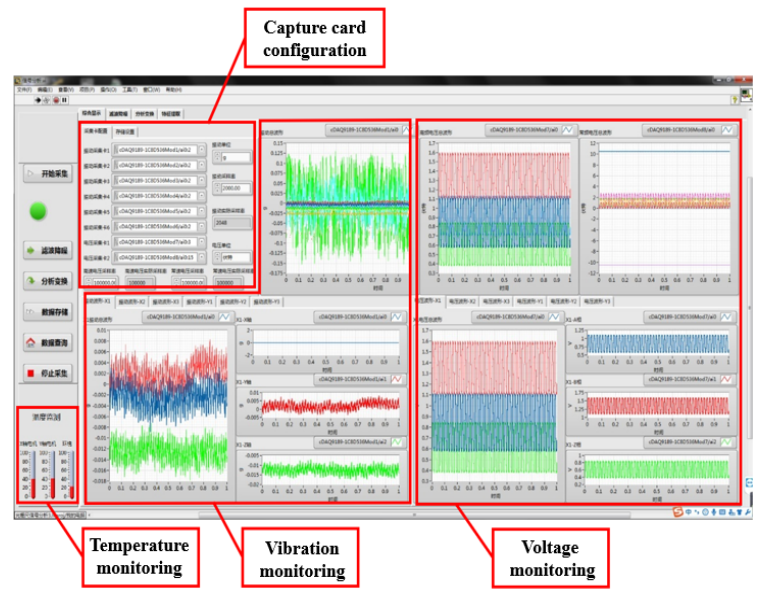

Fig. 4. Condition monitoring system interface.

\subsection{Accuracy measurement scheme of grating ruler}

During the accelerated life test, the measurement accuracy of the grating ruler needs to be checked regularly. If the accuracy exceeds the standard, it is considered that the grating ruler has a degenerate fault. The most important index in the performance parameters of grating rulers are resolution and accuracy. This paper proposes a method for online detection of the accuracy of grating scale based on laser interferometer. The test is suspended periodically, 
and the accuracy of the grating ruler is directly measured by laser interferometer without removing the tested grating rulers.

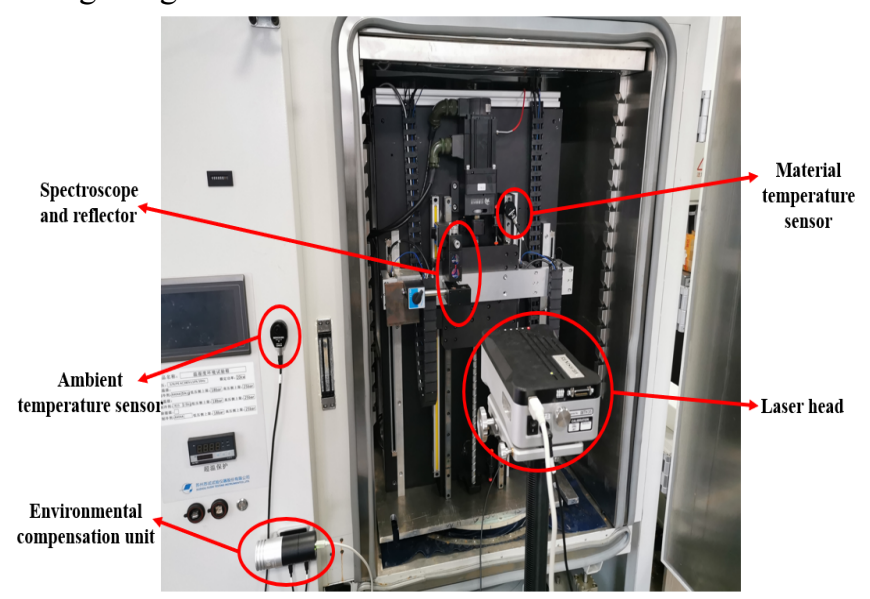

Fig. 5. Accuracy measurement of grating ruler.

The installation and measurement scheme of laser interferometer is shown in Fig 5. The model of the selected laser head is Renishaw XL-80 with measurement accuracy of \pm 0.05 $\mu \mathrm{m}$. The laser head is installed on the tripod through the adjusting bracket, and its position and deflection angle can be adjusted by coarse and fine adjusting nuts. The spectroscope is fixed on the steel rail through the magnetic base. Because the tested grating ruler moves vertically, the spectroscope needs to choose the right-angle form. The reflector is attached to the auxiliary steel plate through a magnetic base, and the auxiliary steel plate is mounted on the linkage aluminum plate by two screws. The linkage aluminum plate drives the three reading heads to move, so as to realize the displacement measurement of the reading head of the tested grating ruler.

\section{Accelerated life test scheme of grating ruler}

\subsection{Step stress accelerated life test scheme}

There are three types of accelerated life test: constant stress accelerated life test, step stress accelerated life test and progressive stress accelerated life test ${ }^{[3-4]}$. Because the grating ruler is a expensive, long-life and precise displacement sensor, the required test cost is too high if constant stress accelerated life test with large sample demand is adopted. In addition, the test system cannot carry out multiple sets of tests with different stress levels at the same time,so the constant stress accelerated life test is not suitable for test. Considering the difficulty of stress loading in the progressive stress accelerated life test and the immaturity of data processing technology, this paper adopts the step stress accelerated life test to test the grating ruler. The schematic diagram of step stress loading is shown in Figure 6. 


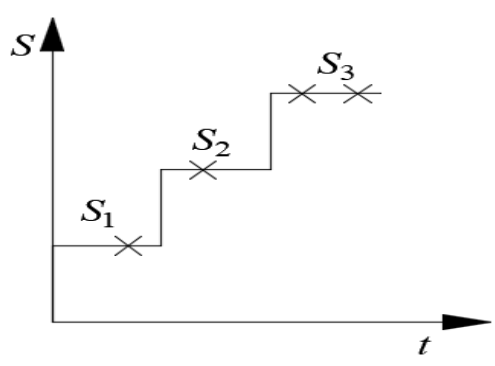

Fig. 6. Schematic diagram of stepping stress loading.

\subsection{Determination of stress loading scheme}

According to the failure mechanism of the grating ruler, the temperature and humidity are used as the main acceleration stress in the accelerated life test,and the velocity stress is applied to simulate the actual condition.

\subsubsection{Temperature and humidity stress loading scheme}

On the basis of the relevant provisions in national standard GB2689. 1-81, the accelerated stress of accelerated life test should be divided into at least four grades. And the maximum and minimum grades cannot exceed the range of the normal working stress of test piece,and the interval between each stress grade should be uniform ${ }^{[5]}$. Therefore, this paper divides the temperature and humidity stress into four grades. The total time of the accelerated life test of the grating ruler is set as $1 / 2$ of the expected mean time between failures of the tested grating ruler under normal working conditions, which is $4500 \mathrm{~h}$. The test time for the four accelerated stress levels is allocated in equal proportion to $1125 \mathrm{~h}$. The final scheme of temperature and humidity stress loading is shown in Table 1.

Table 1. Temperature and humidity stress loading scheme.

\begin{tabular}{cccc}
\hline stress level & $\begin{array}{c}\text { Temperature } \\
T /{ }^{\circ} \mathrm{C}\end{array}$ & $\begin{array}{c}\text { Humidity } \\
R / \%\end{array}$ & $\begin{array}{c}\text { Time } \\
\tau / h\end{array}$ \\
\hline$S_{1}$ & 25 & 45 & 1125 \\
$S_{2}$ & 33.33 & 56.67 & 1125 \\
$S_{3}$ & 41.67 & 68.34 & 1125 \\
$S_{4}$ & 50 & 80 & 1125 \\
\hline
\end{tabular}

\subsubsection{Velocity stress loading scheme}

The maximum speed of the grating ruler in the test is set to $500 \mathrm{~mm} / \mathrm{s}$. According to the relative speed load spectrum of literature ${ }^{[1]}$, the 8 -level load spectrum of speed stress is established in the principle of simulating actual working conditions. During the accelerated life test, the speed stress is cyclically loaded in accordance with the speed, moving distance and moving times given in Table 2 , and temperature and humidity stress are simultaneously loaded. The total moving distance of the grating ruler in a cycle of velocity loading spectrum is $6 \mathrm{~km}$. It takes about $5.4 \mathrm{~h}$ and the average moving speed is about $309 \mathrm{~mm} / \mathrm{s}$ 
Table 2. Grating ruler velocity stress loading spectrum.

\begin{tabular}{ccccc}
\hline Level & Velocity $(\mathrm{mm} / \mathrm{s})$ & Distance $(\mathrm{mm})$ & Times & Proportion(\%) \\
\hline 1 & 50 & 300 & 6800 & 34 \\
2 & 100 & 300 & 3400 & 17 \\
3 & 150 & 300 & 1400 & 7 \\
4 & 200 & 300 & 2200 & 11 \\
5 & 500 & 300 & 200 & 1 \\
6 & 175 & 300 & 1200 & 6 \\
7 & 125 & 300 & 2000 & 10 \\
8 & 75 & 300 & 2800 & 14 \\
\hline
\end{tabular}

\section{Analysis of accelerated life test of grating ruler}

The sample size of grating ruler in the temperature and humidity stress accelerated life test is 6 which is the maximum number that the tested grating rulers can be installed on the servo drive platform, and they are numbered from 1 to 6 in this paper. According to the test scheme, the grating ruler was tested for 4500 hours of accelerated life test.

\subsection{Fault sample data processing}

During the test,the tested grating ruler had 12 faults in total,and the specific fault information is shown in Table 3. Among them, 2 tested grating rulers were scrapped due to the ultra limit of precision, and the remaining 4 tested grating rulers were running until the end of the test.

Table 3. Fault information of grating ruler acceleration life test

\begin{tabular}{ccccc}
\hline $\begin{array}{c}\text { Fault } \\
\text { number }\end{array}$ & $\begin{array}{c}\text { Specimen } \\
\text { number }\end{array}$ & $\begin{array}{c}\text { Stress } \\
\text { level }\end{array}$ & $\begin{array}{c}\text { Fault time } \\
(\mathrm{h})\end{array}$ & Fault mode \\
\hline 1 & 1 & $S_{4}$ & 3681.9 & Accuracy overrun \\
2 & 2 & $S_{3}$ & 2797.1 & Seal damage \\
3 & 2 & $S_{4}$ & 4006.5 & Crack \\
4 & 3 & $S_{2}$ & 1920.3 & Component failure \\
5 & 3 & $S_{4}$ & 3522.0 & Bearing stuck \\
6 & 3 & $S_{4}$ & 4365.1 & Accuracy overrun \\
7 & 4 & $S_{3}$ & 2887.9 & Grating condensation \\
8 & 4 & $S_{4}$ & 4423.8 & Spring loosing \\
9 & 5 & $S_{4}$ & 3606.0 & Component failure \\
10 & 5 & $S_{4}$ & 4289.7 & Component failure \\
11 & 6 & $S_{4}$ & 3614.5 & Seal damage \\
12 & 6 & $S_{4}$ & 3984.8 & Grating condensation \\
\hline
\end{tabular}




\subsection{Analysis of accelerated life distribution model}

Weibull distribution is widely used to describe the life distribution of complex mechatronic products, and it is also applicable to describe the life distribution of the series model of machine fault caused by component fault. The acceleration stress in the grating ruler acceleration life test is temperature and humidity, and the acceleration model is as follows:

$$
\eta=a \exp \left(\frac{b}{T}\right) \exp \left(\frac{c}{R H}\right)
$$

In formula (1), $\eta$ is the characteristic time life of grating ruler under the normal working conditions, $a, b$ and $c$ are undetermined coefficients, $T$ is temperature stress value, and $R H$ is humidity stress value. By using the maximum likelihood estimation algorithm, $a$ is 3.7816 , $b$ is 64.4813 and $c$ is 128.16 , and the characteristic time life of the grating ruler under normal working conditions is 9984 . 26h. The relationship between average time life of grating ruler and temperature and humidity stress is shown in Figure 7.

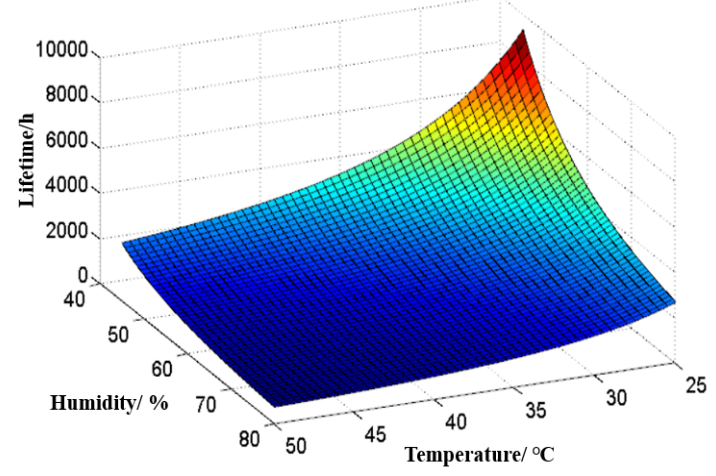

Fig. 7. Diagram of mean time life and temperature and humidity stress.

\section{Conclusion}

An accelerated life test device is designed for the grating ruler, and an accelerated life test scheme based on step stress loading is given. The experimental results show that the designed accelerated life test device can simulate the temperature, humidity and speed stress under the actual working conditions, and the accelerated life analysis model is established based on the test data, which provides a method basis for the life evaluation of the grating ruler. At the same time, the relevant experimental technology in this paper is applied to the innovative practice teaching of undergraduate and graduate students in the way of open and innovative experiments which not only cultivates the students' innovation ability, but also trains their engineering practice skills.

First of all,thank the authors of this paper's references whosework have contributed greatly to the completion of thispaper. In addition, we would like to thank National Science and Technology Major Project (Grant No. 2019ZX04005001009)

\section{References}

1. Song XIN Development of reliability test device for precision grating ruler [D]. Jilin University(2019). 
2. Shijin ZHOU State monitoring and fault diagnosis during reliability test of grating linear displacement sensor[D]. Jilin University (2019).

3. Yichao YIN Research on Reliability Modeling and Analysis Method of Launching Device under Accelerated Life Test and Zero Failure Data[D]. University of Electronic Science and Technology of China(2018).

4. Ranran LUO Research on Accelerated Life Test of Single-phase Smart Meter Reliability[D]. North China Electric Power University(2011).

5. The Fourth Ministry of Machinery Industry of the People's Republic of China. GB2689. 1-81, Constant Stress Accelerated Life Test and General Rules of Accelerated Life Test [S]. Beijing, China Standard Press(1981).

6. JB/T 13686-2019, Grating encoder accelerated life test method [S]. 